\title{
A proposed mechanism for tear film breakup: a molecular level view by employing in-silico approach
}

Lukasz Cwiklik ${ }^{1}$, Adela Melcrova ${ }^{1}$, Philippe Daull², Jean-Sebastien Garrigue²

${ }^{1} \mathrm{~J}$. Heyrovsky Institute of Physical Chemistry, Czech Academy of Sciences, Prague, Czech Republic; ${ }^{2}$ Novagali Innovation Center, Santen SAS, Evry, France

Keywords: dry eye disease, molecular dynamics, molecular simulations, tear film, tear film lipid layer

\section{Background and purpose}

Upon eyelid opening after a blink, tear film (TF) is in a non-equilibrium state and undergoes a complex structural evolution. ${ }^{1}$ In the final stages of this development, the film loses its continuity, which results in so called tear film breakup (TFBU). This phenomenon is critical because it leads to deterioration of the protective role of TF. Importantly, in dry eye disease (DED) the TFBU time is typically shortened according to different patterns, demonstrating that the disease is related to reduced TF stability. ${ }^{2,3}$ The aqueous tear layer at the corneal surface is stabilized by the presence of a relatively thin layer of lipids at the water-air interface, the so-called tear film lipid layer (TFLL). ${ }^{4}$ It has been demonstrated that DED is accompanied by an alteration in TFLL composition, and that TFBU time depends on the properties of TFLL.

Even though TF deterioration and breakup are of key importance for understanding DED, neither the breakup mechanism nor the role played by lipids are understood at a very basic level; such an understanding is required for developing new DED treatments. For instance, it was observed that exogenous and relatively simple surfactant molecules, such as cetalkonium chloride (CKC), are able to stabilize TF in DED in mice and humans. ${ }^{5,6}$ Hence, it would be beneficial to understand the role of natural lipids in TFLL as well as the interactions between its endo- and exogenous components. We hypothesize that various lipid classes of TFLL play

Correspondence: Lukasz Cwiklik, J. Heyrovsky Institute of Physical Chemistry CAS, Dolejskova 2155/3, 18223 Prague, Czech Republic

E-mail: lukasz.cwiklik@jh-inst.cas.cz 
specific roles both during the onset of TF breakup and in maintaining TF stability. We also postulate that, through specific interactions with TF lipids, CKC enhances film stability. ${ }^{7}$ We focus on the behavior of TFLL models deficient in individual lipid components as mimics of DED tear film.

\section{Methods}

In-silico molecular dynamics (MD) simulations are unique in providing molecular-level information regarding organization and dynamics of thin films at interfaces. Here, we simulated lipid films at water-air interface employing a coarse grain MARTINI model. ${ }^{8}$ Such an approach was proven to realistically model TF and TFLL in our earlier studies. ${ }^{9,10}$ Lipid composition of TFLL was approximated by using main lipid classes found in human tears in lipidomics studies. ${ }^{11}$ More specifically, 1-palmitoyl-2-oleoyl-phosphatidylcholine (POPC); 1-palmitoyl-2-oleoyl-phosphatidylethanolamine (POPE); N-palmitoyl-d-erythrosphingosine (PPCE); and N-palmitoyl-d-erythro-sphingosylphosphorylcholine (PPCS) were used as polar lipids in the ratio found in the lipidome of human TF.11 Non-polar lipids were represented by an equimolar mixture of glycerine trioleate (TO) and cholesteryl oleate (CO). Additionally, cations of CKC were incorporated in the lipid film in some of the simulated systems. Lipid films deficient in: polar, non-polar, or both polar and non-polar lipids were considered. The simulation box typically consisted of 6-25 thousand lipids with 90-350 thousand water beads. Lateral sizes between $25 \times 25 \mathrm{~nm}^{2}$ and $34 \times 34 \mathrm{~nm}^{2}$ were considered. MD trajectories of $\sim 2 \mu$ s length were simulated both under equilibrium and non-equilibrium conditions. In the former case, a lipid film completely covering the water surface was equilibrated at the water-air interface and its properties were analyzed. In the non-equilibrium case, initial system configurations were generated from the previous equilibrium simulations by removing half of the already equilibrated lipid film in such a way that the remaining lipids formed a small patch of rectangular shape at the water surface. The resulting MD simulations were hence probing a non-equilibrium process of wetting the water surface by the TFLL.

\section{Results}

We evaluated three main models of TF. First, we considered TF with "non-deficient" TFLL, consisting of such an amount of polar and non-polar lipids that a full lipid film was formed at the water-air interface. In this system, polar lipids resided in a monolayer form with $0.67 \mathrm{~nm}^{2}$ area per lipid, while non-polar lipids formed a multilayer cover atop the polar monolayer. Such a system may be considered a model of relaxed TF, as described in our previous study. ${ }^{9}$ Second, we simulated the 

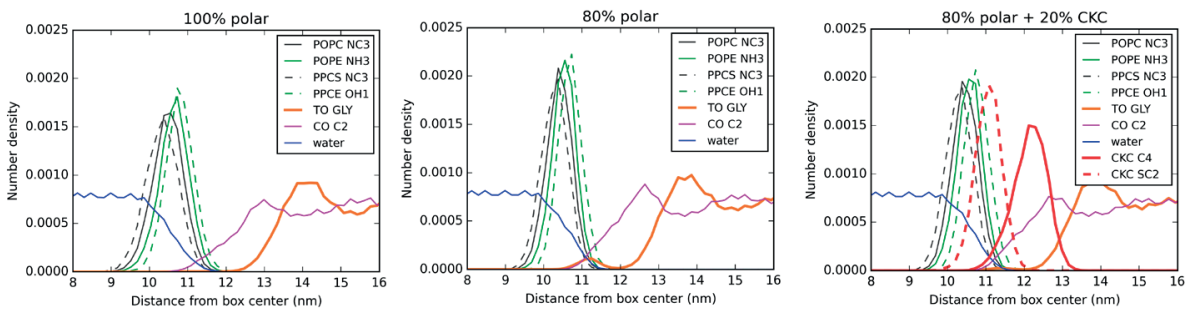

Fig 1. Density profiles calculated for the system with full content of polar lipids (left), the system with $80 \%$ of polar lipids (middle), and the system with $80 \%$ of polar lipids supplemented with CKC (right). The area under each number density is normalized to unity. The density of the choline (POPC, PPCS), amine (POPE), and hydroxyl groups (PPCE) is shown. In TO, the glycerol group is depicted, and the terminus of C20-C27 side chain is shown for CO. In CKC, the terminus of the non-polar chain (C4), as well as the charged headgroup (SC2), are presented.

system with only $80 \%$ polar lipids with regard to the previous model. This can be regarded as polar lipid-deficient TF. Third, the system with $80 \%$ polar lipids was enriched in $20 \%$ (with regard of the initial polar lipid content) of CKC molecules. This model can be viewed as polar lipid-deficient TF supplemented with CKC-containing emulsions. In Figure 1, density profiles corresponding to the three simulated systems are presented. These results were collected in equilibrium MD simulations, i.e., with the lipid film equilibrated at the water-air interface.

In all three models, polar lipids reside at the water-air interface, with their polar groups in contact with water. Hence, polar lipids attain a monolayer-like arrangement. Regarding non-polar lipids, they are spread atop of the polar layer, but their detailed arrangement varies. Namely, in "non-deficient" TFLL, TO molecules penetrate much closer toward polar lipids than the molecules of $\mathrm{CO}$, as evidenced from the partially overlapping density profiles of TO and polar lipids. A further inspection of MD trajectories reveals that there is significant overlap and interdigitation between tails of $\mathrm{CO}$ and tails of polar lipids. At the same time, TO molecules do not overlap with the polar layer and are effectively separated from polar lipids by $\mathrm{CO}$.

In the case of the polar lipids-deficient system, a minor peak at the density profile of TO appears in the region occupied by polar lipids $(\sim 11.2 \mathrm{~nm})$. It indicates that the shortage of polar lipids leads to involvement of some TO molecules in direct contact with their polar counterparts. Inspection of the trajectories show that there are multiple pores formed in the polar monolayer occupied by TO lipids, and hence, contributing to the observed TO-water contacts. Interestingly, when the system is supplemented with CKC, which incorporates in-between polar lipids, the small peak at the TO density profiles virtually disappears. Hence, the presence of CKC leads to diminished TO-polar lipids contact, even in the case of polar lipids deficiency.

In Figure 2, the results of TFLL spreading simulated at the water-air interface are 


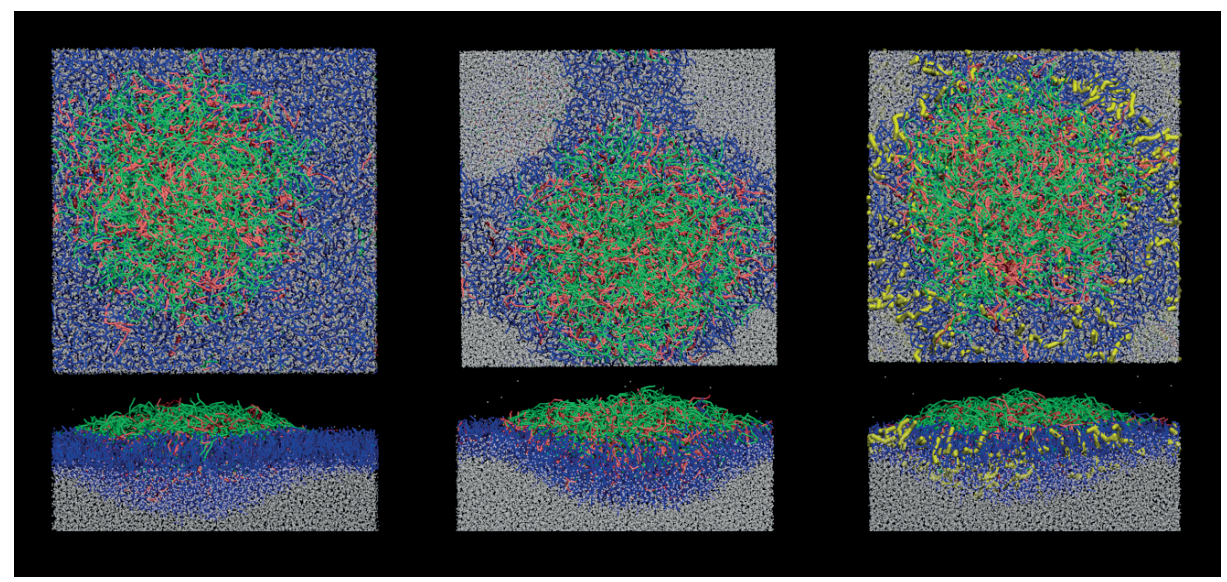

Fig. 2. Representative snapshots of the simulated systems recorded in the final stages of TFLL spreading at the water-air interface in non-equilibrium MD simulations upon equilibration in the non-equilibrium simulations. The system with full content of polar lipids (left), the system with $80 \%$ of polar lipids (middle), and the system with $80 \%$ of polar lipids supplemented with CKC (right) are shown. The first row depicts a view from the gas phase (top view) and the second row presents a cross-section through the middle of the lipid patch (side view). Water is shown in gray, polar lipids in blue, TO in green, CO in brown, CKC in yellow.

presented. In the case of "non-deficient" TFLL, part of the interface is covered by a lipid lens-like multilayer structure resembling the relaxed TFLL, as obtained in our equilibrium cases. At the same time, some of the polar lipids spread homogeneously at the rest of the interface, forming an expanded monolayer. In the case of polar lipids-deficient system, the lens-like multilayer structure is also present. However, the reduced overall amount of polar lipids allows for only partial covering of the interface. Interestingly, the polar lipids are concentrated close to the lens-like structure, not forming an expanded monolayer. In the system supplemented with CKC, the covering of the interface by polar lipids increases, so that CKC effectively promotes spreading of polar lipids in otherwise polar-lipid deficient TFLL.

\section{Conclusions and future perspectives}

The coarse grain MD simulations of the considered TF mimics indicate that when polar lipids are diminished in TFLL, the polar monolayer undergoes poration, leading to enhanced contacts between non-polar triglycerides and water. It can be presumed that, due to unfavorable nature of such non-polar-polar interactions, the lipid film becomes less stable, which can then lead to film breakup. Reduction of polar lipid content also results in less efficient spreading of the lipid film at the water-air interface. The effects of both the unfavorable water-lipids interactions 
and reduced film spreading can be prevented to some extent by the presence of CKC cations. These findings open new perspectives for understanding both TF breakup and spreading mechanisms at the molecular level, as well as for finding new surfactant molecules able to help stabilize TF.

\section{Acknowledgements}

This work was supported by Grant 15-14292S from the Czech Science Foundation. Lukasz Cwiklik and Adela Melcrova have a commercial relationship with Santen SAS (Evry, France).

\section{References}

1. Braun RJ. Dynamics of the tear film. Annu Rev Fluid Mech. 2012;44:267-297.

2. King-Smith PE, Bailey MD, Braun RJ. Four characteristics and a model of an effective tear film lipid layer (TFLL). Ocul Surf. 2013;11:236-245.

3. Yokoi N, Georgiev GA, Kato H, et al. Classification of fluorescein breakup patterns: a novel method of differential diagnosis for dry eye. Am J Ophthalmol. 2017; 180,72-85.

4. Cwiklik L. Tear film lipid layer: A molecular level view. Biochim Biophys Acta-Biomembranes. 2016;10:2421-2430.

5. Quentric Y, Daull P, Feraille L, Elena P-P, Garrigue J-S. Efficacy of a preservative-free cationic emulsion vehicle eye drop in a mouse model of dry eye. Invest Ophthalmol Vis Sci. 2016;57:422.

6. Amrane M, Creuzot-Garcher C, Robert P-Y, et al. Ocular tolerability and efficacy of a cationic emulsion in patients with mild to moderate dry eye disease-A randomised comparative study. J Fr Ophtalmol. 2014;37:589-598.

7. Cwiklik L, Melcrova A, Daull P, Garrigue J-S. Tear film break-up: a molecular level view by employing in silico approach. Invest Ophthalmol Vis Sci. 2017;58:472.

8. Marrink SJ, Risselada HJ, Yefimov S, Tieleman DP, de Vries AH. The MARTINI force field: coarse grained model for biomolecular simulations. J Phys Chem B. 2007;111:7812-7824.

9. Wizert A, Iskander DR, Cwiklik L. Organization of lipids in the tear film: a molecular-level view. Plos One. 2014;9,e92461.

10. Olżyńska A, Cwiklik L. Behavior of sphingomyelin and ceramide in a tear film lipid layer model. Ann Anat. 2017;210:128-134.

11. Rantamaki AH, Seppanen-Laakso T, Oresic M, Jauhiainen M, Holopainen JM. Human tear fluid lipidome: from composition to function. PLoS One. 2011;6:e19553. 